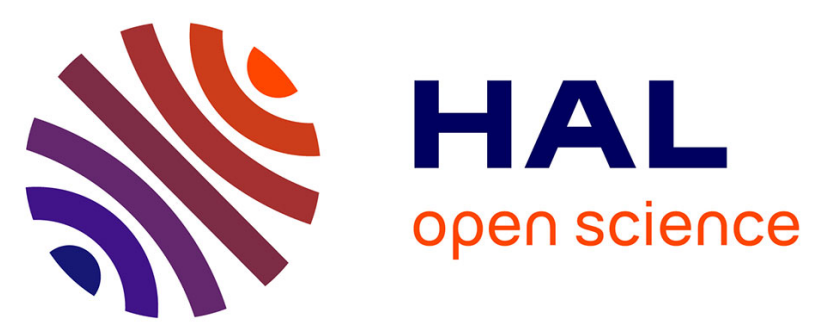

\title{
A fuzzy modeling approach for the long term management of water resource systems
}

Roger Faye, Salam Sawadogo, Felix Mora-Camino, Karim Achaibou

\section{To cite this version:}

Roger Faye, Salam Sawadogo, Felix Mora-Camino, Karim Achaibou. A fuzzy modeling approach for the long term management of water resource systems. FUZZ 2000, 9th IEEE International Conference on Fuzzy Systems, May 2000, San Antonio, United States. pp 499-504, 10.1109/FUZZY.2000.838710 . hal-01022425

\section{HAL Id: hal-01022425 \\ https://hal-enac.archives-ouvertes.fr/hal-01022425}

Submitted on 17 Jul 2014

HAL is a multi-disciplinary open access archive for the deposit and dissemination of scientific research documents, whether they are published or not. The documents may come from teaching and research institutions in France or abroad, or from public or private research centers.
L'archive ouverte pluridisciplinaire HAL, est destinée au dépôt et à la diffusion de documents scientifiques de niveau recherche, publiés ou non, émanant des établissements d'enseignement et de recherche français ou étrangers, des laboratoires publics ou privés. 


\section{A Fuzzy Modeling Approach for the Long Term Management of Water Resource Systems}

\author{
R. M. Faye, S. Sawadogo \\ Ecole Supérieure Polytechnique \\ B.P 10 Thiès-Sénégal \\ (rmfaye,ssalam@ucad.sn)
}

\author{
F. Mora-Camino, A.K. Achaibou \\ LAAS du CNRS, 7 Avenue Colonel Roche \\ 31077 Toulouse-France \\ (mora,achaibou@laas.fr)
}

\begin{abstract}
In this communication, an adaptive solution for the long term management of water resource systems is considered. A fuzzy approach is used to refine the formulation of a recurrent optimization problem attached to the long term
\end{abstract} problem.

Keywords- Modeling, Fuzzy Logic, Water Resource Systems, Intelligent Management Systems.

\section{INTRODUCTION}

In general, the management of water resource systems (WRS) is composed of three levels [2]:

- a strategic level (long term) : it concerns the management of water reserves so as to guarantee availability of the resource and efficiency in its use on an annual basis according to the initial reserves, the climatic conditions, the environmental and technical constraints of the system,

- a tactical level (short term) : it concerns short term resource allocation in accordance with the strategic level to meet expressed demand [3],

- a control level (very short term): it aims to achieve the distribution programs defined at the tactical level.

In the case of long term management, an adaptive optimization approach has been proposed [2] to take into account major uncertainties related to the operations of this kind of system. It consists in readjusting each week the release plan over the whole coming year according to the actual reserves, the last long term demand estimation and the programmed release for the next week. The definition of an optimization objective function is in this case a very intricate question since it involves competition, uncertainty and geographic dispersion. However, it appears to be a crucial point to guarantee the quality of long term management. Fuzzy logic is shown in this communication to be very adequate to refine on-line the formulation of the objective function of the recurrent optimization problem [6] [5]. The proposed approach is illustrated by its application to a case study.

\section{MODELING OF WRS FOR LONG TERM MANAGEMENT}

The structure of a WRS can be represented by an ordered graph (Fig. 1) where the nodes consist of the upstream or intermediate storage dams, canal junctions, exit sections and the arcs that represent the reaches.

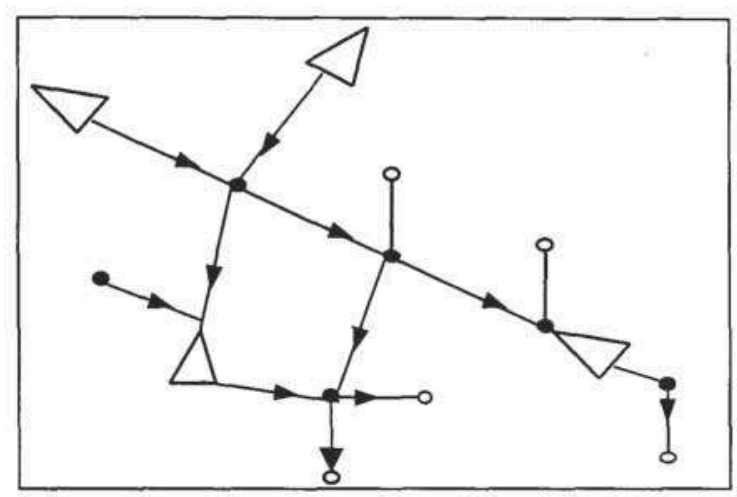

Fig. 1. Example of network representation for a WRS

So, the mathematical model used to manage reserves and supplies on the long term must be able to represent their evolution on a weekly basis. It consists of:

- the evolution of the stock in the storage dams:

$S_{i}(k+1)=\left[1-\rho_{i}(k)\right] S_{i}(k)-Q_{i}(k)-P_{i}(k)-d_{i}(k)+A_{i}(k)$

where: $i \in \mathrm{X}_{\mathrm{B}}$ and

$d_{i}(k)=\max \left\{0, S_{i}^{\max }-\left[\left[1-\rho_{i}(k)\right] S_{i}(k)-Q_{i}(k)-P_{i}(k)+A_{i}(k)\right]\right\}$

- the conservation equation of the resource in the canals:

$\sum_{i \in \Gamma^{-1}} q_{i j}(k)+\sum_{i \in A_{j}} A_{i}(k)=\sum_{i \in \Gamma_{j}} q_{j i}(k)+\sum_{i \in P_{j}} P_{i}(k)$

or $\quad i \in \mathrm{X}_{\mathrm{C}}$

$\sum_{i \in \Gamma_{j}^{-1}} q_{i j}(k)+\sum_{i \in A_{j}} A_{i}(k)=\sum_{i \in \Gamma_{j}} q_{j i}\left(k+p_{i}\right)+\sum_{i \in P_{j}} \mathrm{P}_{i}(k)$

where:

$$
A_{j}(k)=\sum_{i \in \Gamma^{\prime-}} q_{i j}\left(k-p_{i}\right)+a_{j}(k) \quad Q_{j}(k)=\sum_{i \in \Gamma_{j}} q_{j i}(k) \quad j \in \mathrm{X}_{\mathrm{B}}
$$

to which are added the capacity constraints of the flows, stocks, inflows, releases and overflows at various connections.

$q_{u}^{\min } \leq q_{u}(k) \leq q_{u}^{\max } \quad \forall \mathrm{u} \in \mathrm{U}, \quad 0 \leq S_{i}(k) \leq S_{i}^{\max }, \quad A_{i}(k) \geq 0$, $0 \leq P_{i}(k) \leq P_{i}^{\max }, 0 \leq d_{i}(k) i \in \mathrm{X}_{\mathrm{B}} \cup\left\{P_{j}, j \in \mathrm{I}\right\}$ 
where $X_{B}$ is the set of nodes of the intermediate dams, $X_{C}$ the set of nodes of the junction canals, $U$ the set of the arcs or connections, I the set of intermediate pumping station, $S_{i}(k)$ the stock in dam $i$ during period $\mathrm{k}, Q_{i}(k)$ the release volume during the period $k, P_{i}(k)$ the pumping volume during the period $k, A_{i}(k)$ the inflows during period $k, \rho_{i}(k)$ is a factor representing losses (evaporation and infiltration whose mean value is supposed known for the period of considered time), $d_{i}(k)$ the overflow during period $k, q_{j i}(k)$ the input water volume in reach $i$ during period $\mathrm{k}, a_{j}(k)$ the independent inflow for dam $j$ during period $k$, and $p$ is the delay associated to the transfer phenomena and $\Gamma_{j}, \Gamma_{j}^{-1}, P_{j}, A_{j}$ are defined as follows:

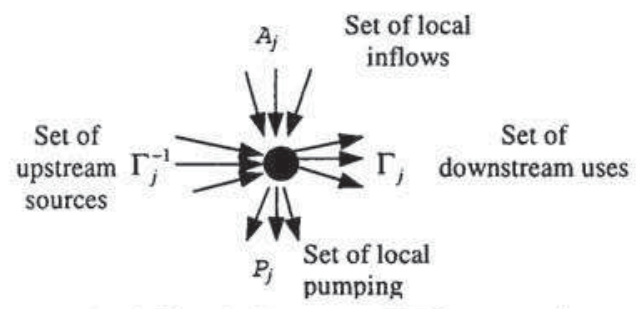

Fig. 2. Flow balance at a WRS connection

\section{PROPOSED APPROACH FOR LONG TERM MANAGEMENT}

Traditionally, long term management considers a diversion curve based on statistical data concerning several years of user demand and upstream inflows. This approach supposes small variations in relation to the mean evolution of the supply and inflows on the long term. In fact, human activities can evolve significantly from one year to another and the meteorology can also present strong changes. So, the approach proposed here is based on an adaptive generation of a weekly release policy through an on-line resolution of a recurrent optimization problem $\left(\mathrm{ROP}_{k}\right)$, whose data are readjusted every week w.r.t new initial conditions for the state of the system and new long term demand and inflows [2]. This approach is illustrated on Fig. 3.

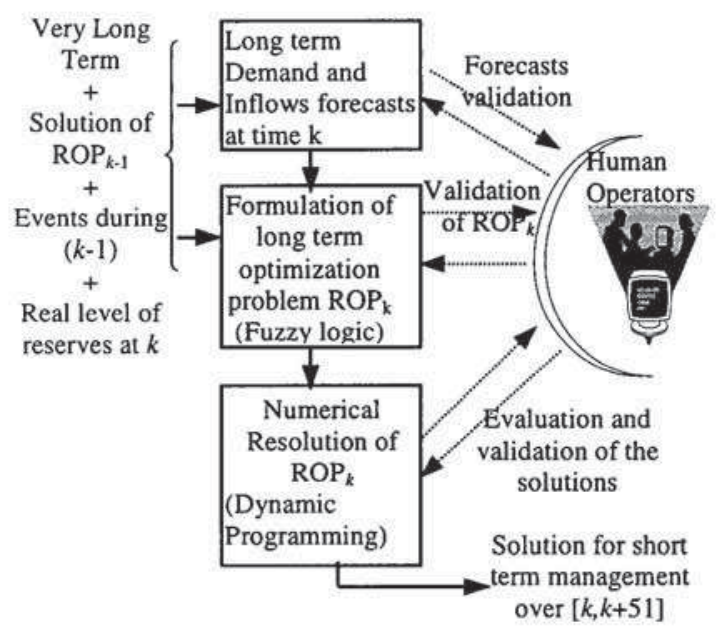

Fig. 3. Long term WRS management structure

\section{FUZZY DEFINITION OF THE RECURRENT OPTIMIZATION PROBLEM}

Owing to the proposed approach in section III, the optimization problem can be formulated in order to make appear at a same time:

- the operational constraints (equation 1 to 3 ),

- the objectives to reach with regard to the satisfaction of the demand and the management of reserves. In this case, it will be about regrouping in an unique criterion the estimation of excesses and deficits of demand.

So, the solution of the optimization problem $\mathrm{ROP}_{k}$, at time $\mathrm{k}$, will give values of weekly volumes to be released by dams so as to minimize, in the year, the deficit between demands and pumpings, considering current available information at the beginning of week $\mathrm{k}$.

This leads to the following formulation compatible with the use of Dynamic Programming technique:

$$
\mathrm{ROP}_{k} \text { Problem: } \min _{Q_{i}^{*}(\lambda) P_{i}^{i}(\lambda)} \sum_{\lambda=k}^{k+51} \sum_{i \in U} \varphi_{u}^{i}\left(D_{i}^{k}(\lambda), P_{i}^{k}(\lambda)\right)
$$

with the constraints:

$$
\begin{aligned}
& S_{i}^{k}(\lambda+1)=\left[1-\rho_{i}(\lambda)\right] S_{i}^{k}(\lambda)-\sum_{j \in \Gamma_{i}} Q_{j}^{k}(\lambda)-P_{i}^{k}(\lambda)-d_{i}^{k}(\lambda)+A_{i}^{k}(\lambda) \\
& \lambda=k \text { to }(k+51), i \in B \\
& S_{i}^{k}(k)=S_{i m}(k) \quad i \in B \\
& \sum_{j \in \Gamma_{i}^{-1}} Q_{j}^{k}(\lambda)+\sum_{i \in A_{i}} A_{i}^{k}(\lambda)=\sum_{j \in \Gamma_{i}} Q_{j}^{k}(\lambda)+\sum_{i \in P_{i}} P_{i}^{k}(\lambda) \quad k \leq \lambda \leq k+51 \\
& 0 \leq Q^{k}(\lambda) \leq Q_{j}^{\max } \mathrm{j} \in \Gamma_{\mathrm{i}}, k \leq \lambda \leq k+51 \\
& S_{i}^{\min } \leq S^{k}(\lambda) \leq S_{i}^{\max } \mathrm{i} \in B, k \leq \lambda \leq k+51 \\
& 0 \leq d_{i}^{k}(\lambda) i \in B, k \leq \lambda \leq k+51 \\
& 0 \leq P^{k}(\lambda) \leq P_{i}^{\max } \quad i \in \underset{j \in / \cup B}{Y} P_{j}, k \leq \lambda \leq k+51 \\
& S_{i}^{k}(k+51) \geq S_{i \min }^{k} i \in B
\end{aligned}
$$

where I is the set of indexes associated to the WRS connections, $P_{j}$ the set of pumpings associated to the connections, $i$ an index representing a group of users, $i \in U$ with $U=Y_{j \in / \cup B} P_{j}, A_{i}$ the set of direct flows contributions to connection $i, B$ the set of indexes associated to the WRS storage dams, $P_{i}^{k}(\lambda)$ the pumped volume by users $i$ during week $\lambda, D_{i}^{k}(\lambda)$ the predicted demand at the beginning of week $k$, for users $i$ during week $\lambda, S_{i}^{k}(\lambda)$ the volume reserve in storage dam $i$ at instant $k$, for the beginning of week $\lambda$, $S_{i \min }^{k}(\lambda)$ is the minimum reserve for dam $i$, in final period end run at $(k+51), Q_{j}^{k}(\lambda)$ the release volume of storage dam $i$ for week $\lambda, A_{i}^{k}(\lambda)$ the volume of contributions to storage dam $i$, predicted at the beginning of week $k$, for week $\lambda, d_{i}^{k}(\lambda)$ the spilled water of storage dam $i$ for week $\lambda$ and $\rho_{i}(\lambda)$ a loss coefficient estimated for the period $\lambda$. 
Constraint (5) describes the evolution of the reserve in dams during a week. Equality (6) imposes the initial condition at instant $k$, where $S_{i m}(k)$ is the reserve measured at the beginning of week $k$. Constraint (7) considers the flow conservation at WRS connections. Inequalities (8), (9), (10), (11) permit to take into account the maximum and minimum levels for variables $Q_{j}, S_{i}, d_{i}, P_{i}$. Constraint (12) considers the minimum storage admitted at the end of the current planning period.

\section{A. Specification of the objective function}

Expression (4) supposes the adoption of a separability hypothesis w.r.t. space (index $i$ ) and time (index $\lambda$ ). It is considered here that supply deficits are directly penalizing for users, while excess of supply, in a scarcity context, is characteristic of waste and inefficiency.

Then function $\varphi_{k \lambda}^{i}\left(D_{i}^{k}(\lambda), P_{i}^{k}(\lambda)\right)$ should satisfy the following properties to penalize concurrently deficits and excesses of supply:

Property 1: $\varphi_{k \lambda}^{i}\left(D_{i}^{k}(\lambda), p\right)$ has a zero minimum at $p=\min \left\{D_{i}^{k}(\lambda), P_{\max }^{i}\right\}$,

Property 2: $\quad \varphi_{k \lambda}^{i}\left(D_{i}^{k}(\lambda), p\right) \quad$ decreases for

$0 \leq p \leq \min \left\{D_{i}^{k}(\lambda), P_{\max }^{i}\right\}$,

Property 3: $\quad \varphi_{k \lambda}^{i}\left(D_{i}^{k}(\lambda), p\right) \quad$ increases $\min \left\{D_{i}^{k}(\lambda), P_{\max }^{i}\right\} \leq p \leq P_{\max }^{i}$.

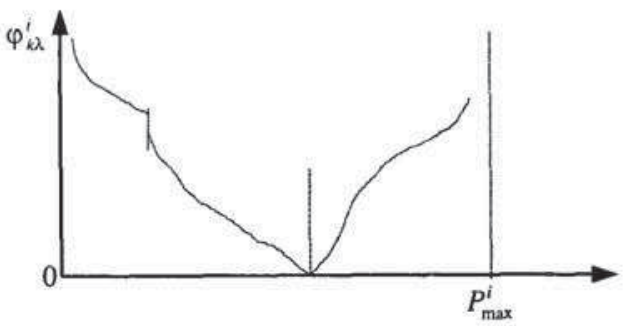

Fig. 4. Example of evolution for function $\varphi$

In fact, segment $\left[0, D_{i}^{k}(\lambda)\right]$ represents the user's side, since users are concerned directly by water deficit, whereas segment $\left[D_{i}^{k}(\lambda), P_{\max }^{i}\right]$ represents the manager's side, since the manager must save reserves (when water is a scarce resource).

However, excesses and deficits have no symmetrical effects on the performance of the system, so unimodal dissymetric function such as:

$$
\begin{aligned}
& \varphi_{k \lambda}^{i}\left(D_{i}^{k}(\lambda), p\right)=\gamma_{i \lambda}^{k} D_{i}^{k}(\lambda)\left[1-\left(p / D_{i}^{k}(\lambda)\right)^{\gamma_{a}^{k}}\right] \\
& \text { if } 0 \leq p \leq D_{i}^{k}(\lambda) \quad \gamma_{i \lambda}^{k} \geq 1
\end{aligned}
$$

$$
\begin{aligned}
& \varphi_{k \lambda}^{i}\left(D_{i}^{k}(\lambda), p\right)=\beta_{i \lambda}^{k} D_{i}^{k}(\lambda)\left[\left(p / D_{i}^{k}(\lambda)\right)^{\beta_{a}^{k}}-1\right] \\
& \text { if } D_{i}^{k}(\lambda) \leq p \leq P_{\max }^{i} \beta_{i \lambda}^{k} \geq 1
\end{aligned}
$$

which depend only on one parameter to be determined ( $\gamma_{i \lambda}^{k}$ or $\beta_{i \lambda}^{k}$ ), appear to be good candidates to express the planning goals.

\section{B. Fuzzy determination of the weighting parameters}

In this section, it is shown how fuzzy modeling can be used to establish relevant weightings for the objective function of the long term WRS management problem [2]. Here the proposed approach is illustrated in the case of the penalization of supply excesses.

A first approach would consist in comparing at instant $k$, the amount of supply determined at instant $(k-1)$ for the next week, to the sum of demands for the same period. However, this approach brings back the global deficit (or excess) to the exit section of the storage dams and therefore makes abstraction of the structure of the managed system. In fact, excesses are only critical because there is, elsewhere or in other future periods, some supply deficit. It appears therefore, that degree of criticity of excesses is related to the supply deficit.

A simple measure of the so-called "relative operational deficit" which takes into account the structure of the distribution system and the way the system is operated can be defined as:

$$
d f^{k}=\left(\sum_{\lambda=k}^{k+50} \sum_{i \in B} d f_{i}^{k}(\lambda)\right) /\left(\sum_{\lambda=k}^{k+50} \sum_{i \in B} D_{i}^{k}(\lambda)\right)
$$

It seems also necessary to take into account the tendency of the deficit: if it increases from one period to another, it will be necessary to anticipate its future effect on demand by increasing the corresponding weighting parameter. The procedure will be the inverse if this tendency decreases. This tendency must be calculated over a period of time which takes into account seasonal effects or vegetative cycles associated to cultures. A period of one month seems to be acceptable. This period can be adapted according to the climatic and agricultural features of the region under operation.

A tendency index of the deficit can be given by the expression:

$$
d d f^{k}=\left[\sum_{\lambda=k-p}^{k} d f^{\lambda} \lambda-\sum_{\lambda=k-p}^{k} d f^{\lambda} \sum_{\lambda=k-p}^{k} \lambda\right] /\left[\sum_{\lambda=k-p}^{k} \lambda^{2}-(p+1) \sum_{\lambda=k-p}^{k} \lambda\right](16)
$$

which corresponds to an evaluation of the medium slope of the "relative operational deficit" on its $(p+1)$ last periods of time by the least simple square method.

Therefore, the operational deficit and its tendency can be valued according to the linguistic variables associated to the following fuzzy linguistic values: 
VW: "Very Weak", W: “Weak", M: "Medium", H: "High", VH: "Very High", EH: "Excessively High",

Z: "Zero", NH: "Negative Large", NM: "Negative Medium", NS: "Negative Small",

PS: "Positive Small", PM: "Positive Medium", PH: "Positive Large".

A possible linguistic evaluation of the deficit and its tendency are displayed in Fig. 5 and Fig. 6:

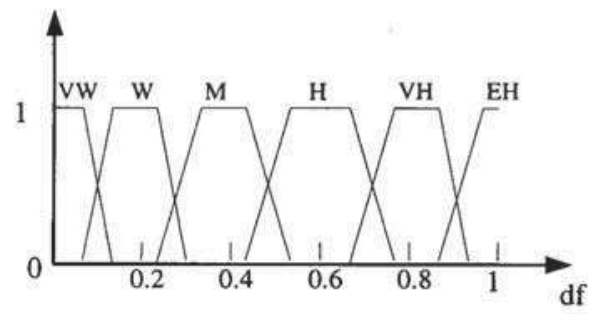

Fig. 5. Linguistic evaluation of the deficit

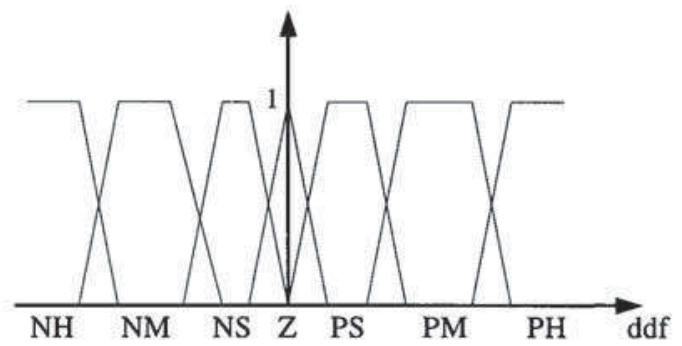

Fig. 6. Linguistic evaluation of the tendency of the deficit

A base of rules can be developed according to expert knowledge. This leads to a small size base of rules (TABLE I),

TABLE I

EXAMPLE OF A BASE OF RULES ASSOCIATED TO THE WEIGHTING PARAMETERS OF SUPPLY EXCESS

\begin{tabular}{|c|c|c|c|c|c|c|}
\hline$d d f d f$ & $V W$ & $W$ & $M$ & $H$ & $V H$ & $E H$ \\
\hline$N H$ & VS & VS & $\mathrm{S}$ & $\mathrm{M}$ & $\mathrm{M}$ & $\mathrm{M}$ \\
\hline$N M$ & $\mathrm{VS}$ & $\mathrm{VS}$ & $\mathrm{S}$ & $\mathrm{M}$ & $\mathrm{M}$ & $\mathrm{M}$ \\
\hline$N S$ & $\mathrm{~S}$ & $\mathrm{~S}$ & $\mathrm{~S}$ & $\mathrm{M}$ & $\mathrm{M}$ & $\mathrm{H}$ \\
\hline$Z$ & $\mathrm{~S}$ & $\mathrm{~S}$ & $\mathrm{M}$ & $\mathrm{M}$ & $\mathrm{H}$ & $\mathrm{H}$ \\
\hline$P S$ & $\mathrm{M}$ & $\mathrm{M}$ & $\mathrm{M}$ & $\mathrm{M}$ & $\mathrm{H}$ & $\mathrm{H}$ \\
\hline$P M$ & $\mathrm{M}$ & $\mathrm{M}$ & $\mathrm{M}$ & $\mathrm{H}$ & $\mathrm{VH}$ & $\mathrm{VH}$ \\
\hline$P H$ & $\mathrm{H}$ & $\mathrm{H}$ & $\mathrm{H}$ & $\mathrm{VL}$ & $\mathrm{VH}$ & $\mathrm{VH}$ \\
\hline
\end{tabular}

associated to a fuzzy partition of the field of values of the weighting parameter $\beta$ (Fig.7).

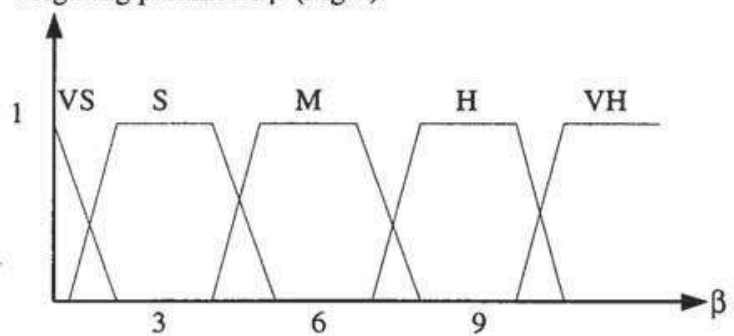

Fig. 7. Fuzzy partition of the field values for the weighting parameter $\beta$ where

VS: "Very Small", S: "Small", M: "Medium", H: "High", VH: "Very high".

Fuzzy rules are of the form: "If $d d f$ is NS and $d f$ is $\mathrm{H}$ then $\beta$ is $\mathbf{M}$ ". For example, a relative operational deficit of $30 \%$ and a deficit tendency of +0.4 , using Mamdani method of inference with a center of gravity of defuzzification [1] lead to $\beta=6$.

\section{APPLICATION}

The proposed approach has been applied to different WRS. Here its application to a dual WRS, dedicated to irrigation, is displayed to illustrate its practicability.

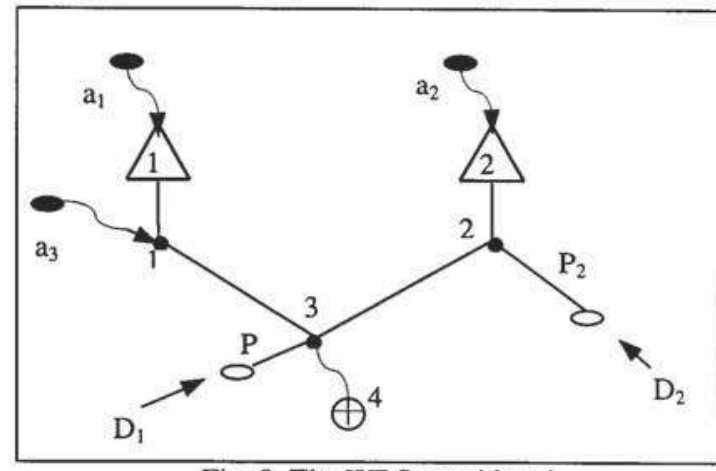

Fig. 8. The WRS considered

The WRS is characterized by the following parameters:

$S_{1}^{\max }=10^{7} \mathrm{~m}^{3}, S_{2}^{\max }=0.510^{7} \mathrm{~m}^{3}, S_{1}^{\min }=210^{6} \mathrm{~m}^{3}$, $S_{2}^{\min }=0.510^{6} \mathrm{~m}^{3}, S_{1 m}=2.510^{6} \mathrm{~m}^{3}, S_{2 m}=0.710^{6} \mathrm{~m}^{3}$, $q_{11}^{\max }=0.710^{6} \mathrm{~m}^{3} /$ week, $q_{13}^{\max }=0.710^{6} \mathrm{~m}^{3} /$ week, $q_{22}^{\max }=0.710^{6} \mathrm{~m}^{3} /$ week, $q_{23}^{\max }=0.310^{6} \mathrm{~m}^{3} /$ week, $q_{34}^{\max }=0.710^{6} \mathrm{~m}^{3} /$ week, $q_{34}^{\min }=0.28610^{6} \mathrm{~m}^{3} /$ week, $P_{1}^{\max }=1.3410^{6} \mathrm{~m}^{3} /$ week, $P_{2}^{\max }=0.67210^{6} \mathrm{~m}^{3} /$ week $\varepsilon_{1}=0.17510^{6} \mathrm{~m}^{3}, \varepsilon_{2}=0.16510^{6} \mathrm{~m}^{3}, \varepsilon_{3}=0.12510^{6} \mathrm{~m}^{3}$, $\rho_{1}=\rho_{2}=0.003$.

The long term demands are such as:

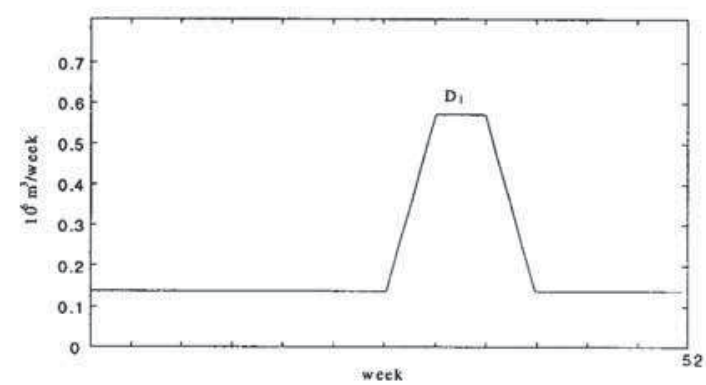

Fig. 9. Long term demand at station 1 


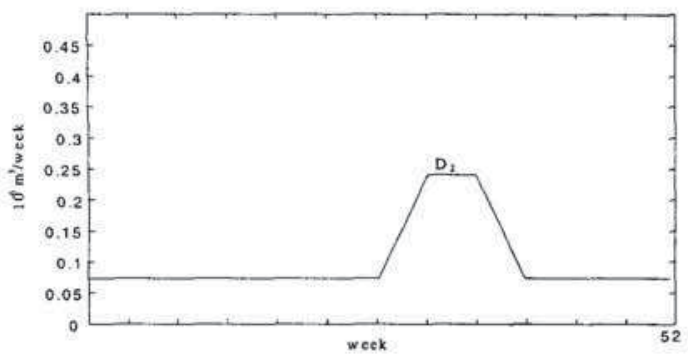

Fig. 10. Long term demand at station 2

The sequential structure of the constraints, the separable structure and the non convexity of the criterion lead to the consideration of the Dynamic Programming technique as a potential method for the resolution of the recurrent optimization problem [4].

It is assumed here that in the recent past, no excesses situation has been observed. Therefore, a possible excess must be strongly penalized. As week 1 is in an inter-seasonal worker period ( between the end of the irrigation period and the beginning of the new raining season), here $d d f^{d}=0$ and $d f^{d}$ is roughly assumed equal to 0.3 . Using table I and Mamdani's method of inference with a center of the gravity method of defuzzification, the value of $\beta^{\prime}$ obtained is approximately 4 . An example of the evolution of the resulting weighting parameter $\beta$ over the next year is given in fig. 11 .

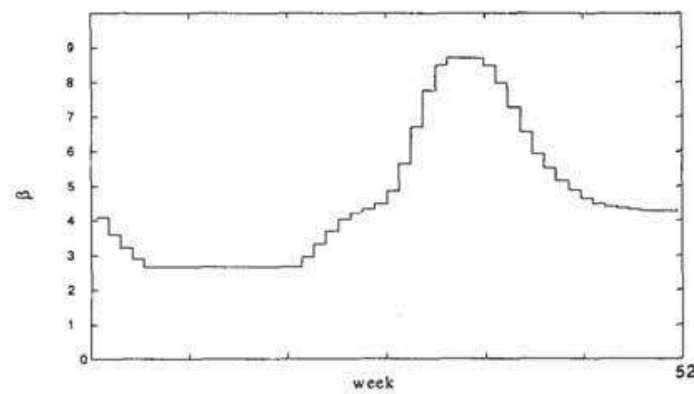

Fig. 11. Estimation of $\beta^{k}$ over the next year at week 1

The resolution of the $\mathrm{ROP}_{\mathrm{k}}$ by Dynamic Programming provides the following long term management plan:

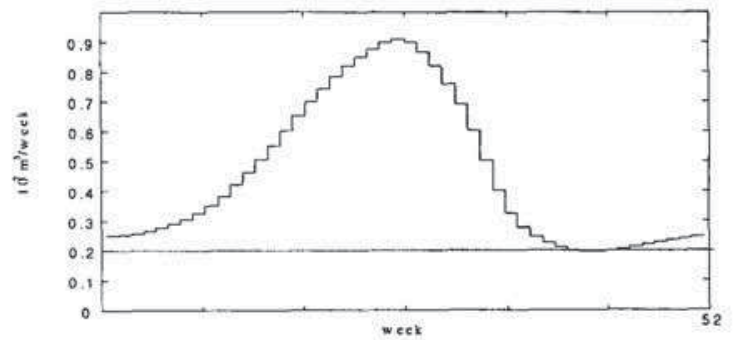

Fig. 12. Long term evolution of the reserve of dam 1

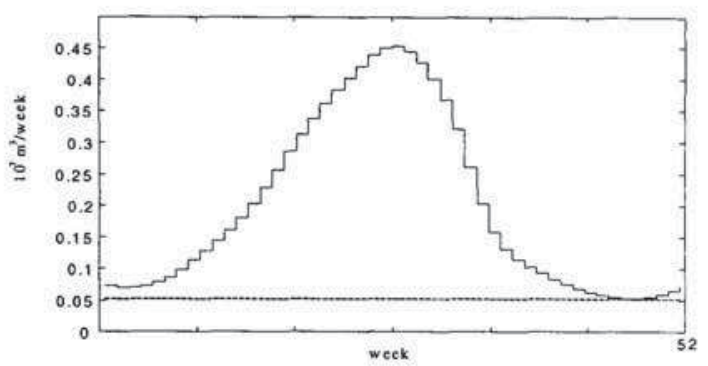

Fig. 13. Long term evolution of the reserve of dam 2

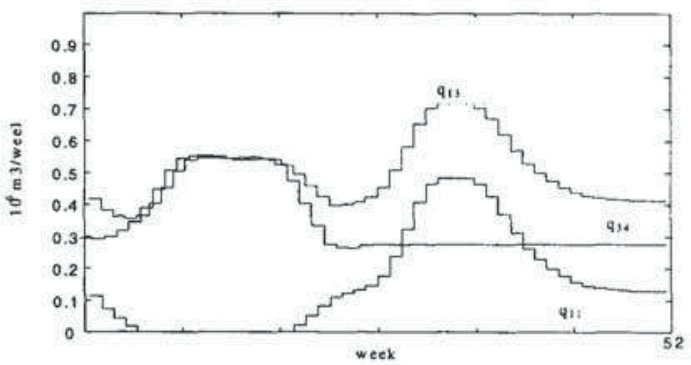

Fig. 14. Long term evolution of the discharge associated to side 1

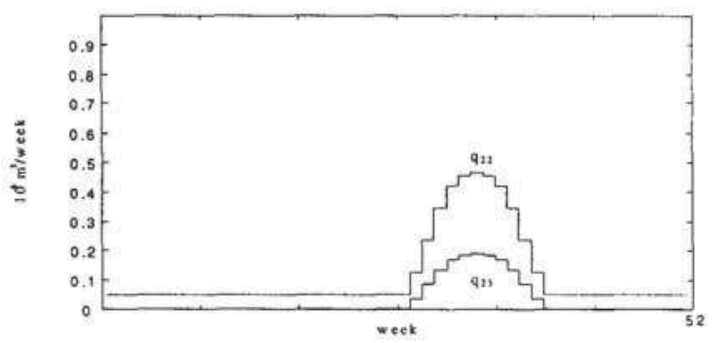

Fig. 15. Long term evolution of the discharge associated to side 2

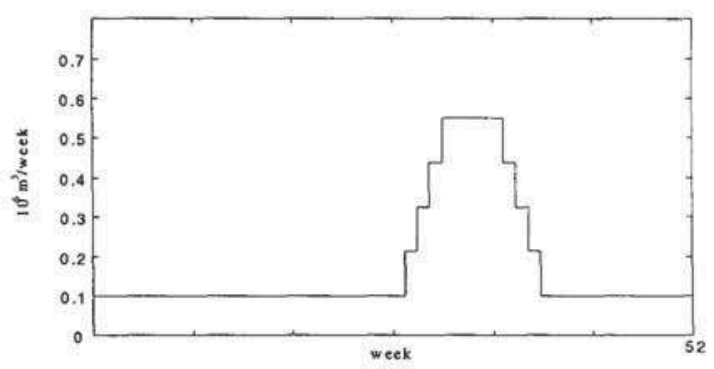

Fig. 16. Long term evolution of pumping $P_{1}$ 


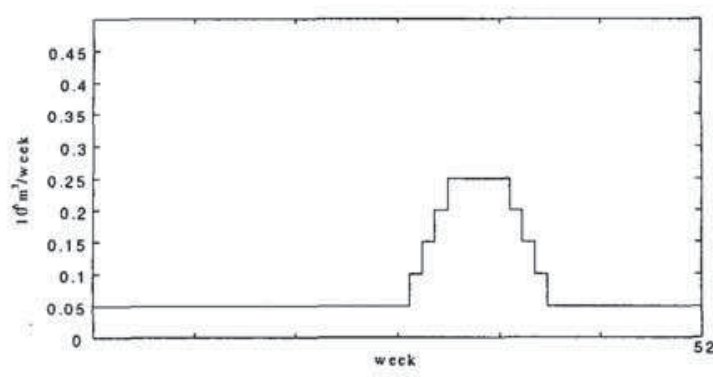

Fig. 17. Long term evolution of pumping $\mathrm{P}_{2}$

\section{CONCLUSION}

The proposed approach applied to the long term management of a WRS is based on the formulation of a recurrent optimization problem whose criterion must penalize discrepancies between offers and demands over time and space. This leads to the problem of the on-line definition of a set of weightings for the adopted criterion function. In this communication it has been shown how to achieve this task using Fuzzy Logic. It is clear that this technique leads to a practical adaptive solution for the modeling of the recurrent optimization problem $\left(\mathrm{ROP}_{k}\right)$ considering that it takes into account the operational constraints of the system and the objectives to reach with regard to the satisfaction of long term demand and management of reserves.

\section{REFERENCES}

[1] D. Dubois, H. Prade, Fuzzy sets and systems, Academic Press, 1980

[2] R.M. Faye, Une Approche Intégrée pour la Gestion des Ressources en Eau faisant appel aux Techniques Floues et Neuronales, Ph.D Thesis, University Paul Sabatier Toulouse, $N^{\circ} 3399$, LAAS Report $N^{\circ} 99261,14$. June (1999).

[3] R.M. Faye, F. Mora-Camino, S. Sawadogo, and S. Gonzalez-Rojo, Flexible Management of Water Resource Systems, IEE $15^{\text {th }}$ International Conference on CAD/CAM, Robotics and Factories of the Future, vol. 1, pp. 7-12, August 1999, Aguas de Lindoia, SP Brazil.

[4] M. S. Hajilal, N. H. Rao, P. B. S. Sarma, Real time operation of reservoir based canal irrigation systems, Agricultural Water Management, vol. 38, pp. 103-122, 1998.

[5] K. Hirota, M. Sugeno, Industrial Applications of Fuzzy Technology in the World, Advances in Fuzzy Systems-Applications and Theory, vol. 2, World Scientific Publishing, 1995.

[6] L. A. Zadeh, Outline of a new approach to the analysis of complex Systems and Decision Processes. IEEE Trans. on Systems Management and Cybernetics, vol. SMC-3, pp. 28-44, 1973. 